\title{
On the sulutions of a fractional differemtial equation
}

\author{
Runqing Cui
}

College of Mathematics and Informatics, Henan Polytechnic University, Jiaozuo 454003, China Igdcuirunqing@yeah.net

\section{Abstruct}

We have showed the results obtained in [1] are incorrect and the fractional complex transform is invalid to the fractional differential equation which contain modified Riemann-Liouville fractional derivative.

\section{Keyword:}

fractional complex transform; fractional differential equation; modified Riemann-Liouville fractional derivative.

\section{INTRUDUCTION}

Fractional differential equations are generalizations of classical differential equation. During the past decades, fractional differential equations appeared more and more frequently in various research areas of science and engineering [1]. Therefore, many authors want to find the exact analytical solutions or approximate analytical solutions of some fractional differential equations using different ways [2].

In [1], by using fractional complex transform, Zhang et al. studied the following frctional differential equation:

$$
\partial_{t}^{\alpha} u(x, t)=k \partial_{x}^{\beta} u(x, t), \quad t \in R^{+}, x \in R
$$

Subject to the initial condition

$$
u(x, 0)=2 x
$$

where $k$ is a positive coefficient, $0<\alpha<1,0<\beta<1, u(x, t)$ is the real-valued variable function, $\partial_{t}^{\alpha}$ and $\partial_{x}^{\beta}$ are modified Riemann-Liouville fractional derivatives.

They obtained the following exact solution to the initial problems (1) and (2):

$$
u(x, t)=2\left(\frac{\Gamma(1+\beta)}{p}\right)^{\frac{1}{\beta}}\left(\frac{p x^{p}}{\Gamma(1+\beta)}+\frac{q t^{\alpha}}{\Gamma(1+\alpha)}\right)^{\frac{1}{\beta}}
$$

where $p$ and $q$ are free parameters satisfying $k p-q=0$.

In this note, we will showed that above solution (3) is not solution of the problem (1)-(2) by counterexample, and therefore we can assert the fractional complem transform is false to the initial problem.

\section{Main result}

We first recall the dedinition of modified Riemann-Liouville fractional derivatives.

Dedinition: Let $f(x)$ is a continuous but not necessarily differentiable function, then its fractional derivative of order $\alpha(0<\alpha<1)$ is defined by the following expression [2]: 


$$
\partial^{\alpha} f(t)=\frac{1}{\Gamma(1-\alpha)} \frac{d}{d t} \int_{0}^{t}(t-\xi)^{-\alpha}(f(\xi)-f(0)) d \xi .
$$

From the above Definition, we can check the results obtaind in [1].

For simplicity, we take $\alpha=\frac{1}{3}, \beta=\frac{1}{2}, k=1$, and $p=q=1$.

Thus the initial problem (1)-(2) becomes

$$
\left\{\begin{array}{c}
\partial^{1 / 3} u(x, t)=\partial^{1 / 2} u(x, t) \\
u(x, 0)=2 x
\end{array}\right.
$$

and the solution (3) becomes

$$
u(x, t)=2 x+\frac{4 \Gamma\left(\frac{3}{2}\right)}{\Gamma\left(\frac{4}{3}\right)} x^{\frac{1}{2}} t^{\frac{1}{3}}+\frac{2 \Gamma^{2}\left(\frac{3}{2}\right)}{\Gamma^{2}\left(\frac{4}{3}\right)} t^{\frac{2}{3}} .
$$

But, by the formula (4), we have

and

$$
\partial_{t}^{\frac{1}{3}} u(x, t)=2 \Gamma\left(\frac{1}{2}\right) x^{\frac{1}{2}}+\frac{9 \Gamma^{2}\left(\frac{1}{2}\right) \Gamma\left(\frac{2}{3}\right)}{\Gamma^{3}\left(\frac{1}{3}\right)} t^{\frac{1}{3}}
$$

$$
\partial_{x}^{\frac{1}{2}} u(x, t)=\frac{4}{\Gamma\left(\frac{1}{2}\right)} x^{\frac{1}{2}}+\frac{3 \Gamma^{2}\left(\frac{1}{2}\right)}{\Gamma\left(\frac{1}{3}\right)} t^{\frac{1}{3}}
$$

From the above two expressions, we obtain :

$$
\partial_{t}^{\frac{1}{3}} u(x, t) \neq \partial_{x}^{\frac{1}{2}} u(x, t) .
$$

This shows that the function (6) does not the solution of the problem (5). Moreover, we see that the function (3) does not the solution of the problem (1)-(2), so that the fractional complex transform is invalid to the fractional differential equation which contain modified Riemann-Liouville fractional derivative.

\section{Conclusion}

We have showed the results obtained in [1] are incorrect and the fractional complex transform Is invalid to the problem (1)-(2). 


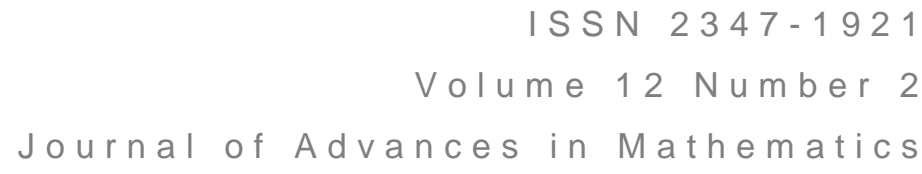

\section{References}

1. Li Z B, He J H. Fractional complex transform for fractional differential equations[J]. Math. Comput. Appl, 2010, 15(5): $970-973$.

2. Jumarie G. Modified Riemann-Liouville derivative and fractional Taylor series of nondifferentiable functions further results[J]. Computers \& Mathematics with Applications, 2006, 51(9): 1367-1376.

3. Diethelm K, Ford N J. Analysis of fractional differential equations[J]. Journal of Mathematical Analysis and Applications, 2002, 265(2): 229-248.

4. Ray S S, Bera R K. An approximate solution of a nonlinear fractional differential equation by Adomian decomposition method[J]. Applied Mathematics and Computation, 2005, 167(1): 561-571.

5. Shawagfeh N T. Analytical approximate solutions for nonlinear fractional differential equations[J]. Applied Mathematics and Computation, 2002, 131(2): 517-529.

6. $\mathrm{Li} \mathrm{Z} \mathrm{B,} \mathrm{He} \mathrm{J} \mathrm{H.} \mathrm{Application} \mathrm{of} \mathrm{the} \mathrm{fractional} \mathrm{complex} \mathrm{transform} \mathrm{to} \mathrm{fractional} \mathrm{differential} \mathrm{equations[J].} \mathrm{Nonlinear}$ Science Letters A-Mathematics, Physics and Mechanics, 2011. 